\title{
Inventaire Et Gestion Des Plantes Médicinales Dans Quatre Localités Du Niger
}

\author{
Soumaila Mounkaila \\ Université Abdou Moumouni de Niamey, Faculté des Sciences et \\ Techniques, Département de Biologie Laboratoire Garba Mounkaila, \\ Niamey, Niger. \\ Université d'Agadez, Faculté des Sciences et Techniques; Agadez, Niger.
}

\section{Barmo Soukaradji}

Institut National des Recherches Agronomiques du Niger.

\author{
Boube Morou \\ Saley Karim \\ Hassane Bil-Assanou Issoufou
}

Université de Maradi, Niger.

\section{Ali Mahamane}

Université de Diffa, Niger.

\section{Kalid Ikhiri}

Université Abdou Moumouni de Niamey, Faculté des Sciences et

Techniques, Département de Chimie, Niamey Niger.

\section{Mahamane Saadou}

Université Abdou Moumouni de Niamey, Faculté des Sciences et

Techniques, Département de Biologie Laboratoire Garba Mounkaila, Niamey, Niger.

\begin{abstract}
Objective: the present study, aims to list medicinal plants, and gathering the maximum of information concerning the therapeutic uses practised by the population and finally to determine the impact of the takings of the various organs on the survival of plants. Methodology and results: An ethnobotanic survey was driven in four municipalities in Niger, to list healing plants used by the populations. The study showed that 111 species being of 84 kinds and 48 families are used in the traditional pharmacopoeia. The main listed families are Caesalpiniaceae, Combretaceae, Fabaceae, Euphorbiaceae, Anarcadiaaceae and Rubiaceae, with respectively, 10, 7, 7, 6, 5 and 4 species. For fragments, the most used organs are sheets, barks and roots. The results of the study also show that the majority of remedies are
\end{abstract}


prepared in form of decoction. The taking of organs especially roots are not without consequence for the survival of the medicinal plants according to the local population. Conclusion and research application: These results can be used for scientific research in phytochemistry and in pharmacology to go towards the development of improved traditional medicine.

Keywords: Ethnobotanics, Healing Plants, Niger

\section{Resume}

Objectif : La présente étude, a pour objectif de recenser les plantes médicinales, de réunir le maximum d'informations concernant les usages thérapeutiques pratiqués par la population et enfin de déterminer l'impact des prélèvements des différents organes sur la survie des plantes. Méthodologie et résultats : Une enquête ethnobotanique a été conduite dans quatre communes du Niger. L'étude a montré que 110 espèces relevant de 89 genres et de 47 familles sont utilisées dans la pharmacopée traditionnelle. Les principales familles recensées sont les Fabaceae, les Combretaceae, les Euphorbiaceae, les Anarcadiaceae et les Rubiaceae, avec respectivement, 26, $6,5,5$ et 4 espèces. Les fragments ou organes les plus utilisés sont les feuilles, les écorces et les racines. Les résultats de l'étude ont aussi montré que les remèdes sont préparés principalement sous forme de décoction. Le prélèvement des organes surtout les racines contribue à la mort des espèces médicinales selon la population. Conclusion et application de la recherche : Ainsi, ces résultats peuvent être utilisés pour les recherches scientifiques en phytochimie et en pharmacologie afin d'aller vers la mise au point des médicaments traditionnels améliorés.

Mots clés : Ethnobotanique, Plantes médicinales, Niger.

\section{Introduction}

Durant des siècles, nos ancêtres utilisent les plantes pour soulager leurs douleurs, guérir leurs maux et panser leurs blessures. De génération en génération, ils ont transmis leur savoir et leurs expériences simples en s'efforçant quand ils le pouvaient de les consigner par écrit. Malgré le progrès de la pharmacologie, l'usage thérapeutique des plantes médicinales est très présent dans certains pays du monde et surtout les pays en voie de développement, en l'absence d'un système médical moderne (Tabuti et al., 2003). Les plantes médicinales sont utilisées dans tous les pays. Dans les pays développés, elles fournissent, dans la plupart des cas, la matière première pour la préparation industrielle des dérivés chimiques purs et spécialités pharmaceutiques. Dans beaucoup d'autres pays en développement, elles sont utilisées sous forme brute d'extraits ou d'infusés 
servant de base à presque toutes les formes galéniques et thérapeutiques (Dasylva, 2001).

Au Sahel, comme dans la plupart des pays en voie de développement, la pharmacopée traditionnelle joue un rôle important en milieu rural (Yamba, 1993). En raison de l'enclavement des zones rurales, des infrastructures sanitaires inexistantes ou rudimentaires, du coût élevé des préparations pharmaceutiques, des faibles revenus, les plantes médicinales constituent un atout majeur pour les populations rurales (Dibong et al., 2011). Ainsi, on ne va au dispensaire qu'en dernier recours lorsque la médecine traditionnelle s'est révélée inefficace (Manzo, 1996). Selon l'OMS (2002), 80\% de la population utilisent la médecine traditionnelle à base des plantes médicinales dans les soins de santé primaire. Cela justifie que l'intérêt et la recherche dans le domaine du savoir indigène aient augmenté ces dernières années, spécialement après la Conférence de Rio de Janeiro (Wezel, 2002).

Les plantes médicinales constituent un patrimoine précieux pour l'humanité et plus particulièrement pour la majorité des communautés démunies des pays en voie de développement qui en dépendent pour assurer leurs soins de santé primaires et leurs subsistances (Salhi et al., 2010). La médecine traditionnelle constitue pour l'Afrique en général et pour le Niger en particulier un patrimoine culturel et économique d'une importance incontestable. Cependant la mise en valeur de ce patrimoine pose un certain nombre de problèmes dont un des plus importants demeure la méfiance des guérisseurs à livrer leurs connaissances souvent initiatiques (Ikhri et al., 1984). Et selon Ouôba et al., (2006) le risque de voir disparaître certains guérisseurs sans avoir livré leurs secrets, amène à redoubler d'efforts pour conserver par écrit les informations sur les plantes médicinales mais aussi, pour leur valorisation. C'est dans cette optique qu'une étude ethnobotanique a été conduite au Niger dont l'objectif consiste à recenser les plantes médicinales et évaluer l'impact des prélèvements sur leur survie.

\section{Matériel et méthodes}

\section{Milieu d'étude}

L'étude a été menée dans les communes de Gaya, Tanda, Tamou, et la communauté urbaine de Niamey (Figure 1). 


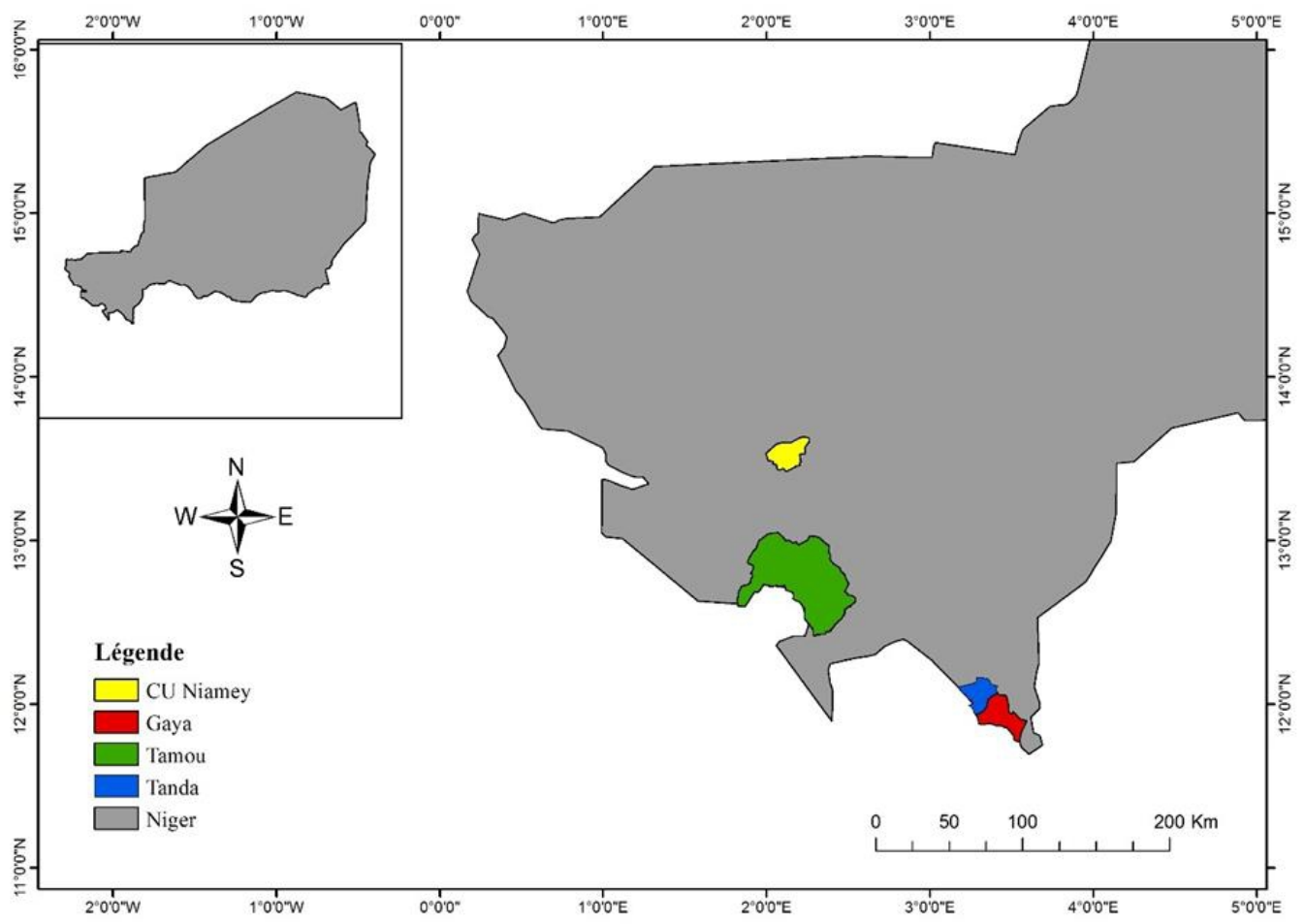

Figure 1 : Localisation des sites d'étude

La ville de Niamey est située entre $13^{\circ} 31$ de latitude Nord et $2^{\circ} 26$ de longitude Est. Elle est construite sur deux plateaux surplombant le fleuve Niger, à $218 \mathrm{~m}$ d'altitude. Le climat est de type sahélien caractérisé par une saison pluvieuse (4 à 5 mois) et une saison sèche plus longue que la précédente, avec une pluviométrie variant de 500 à $750 \mathrm{~mm}$ par an (Mounkaila et al., 2015). La végétation est caractérisée par des fourrés composés principalement des espèces du genre Combretum sur les plateaux latéritiques et des steppes sur les terrasses sableuses, dans les vallées sèches et sur les dunes (Yacouba, 1999).

Les communes de Gaya et Tanda sont localisées dans le département de Gaya (région de Dosso). La commune de Gaya est située à l'Est de celle de Tanda et est située entre $3^{\circ} 10^{\prime} 35^{\prime \prime}$ et $3^{\circ} 37^{\prime} 48^{\prime \prime}$ de longitude Est et $11^{\circ} 48^{\prime} 24^{\prime \prime}$ et $12^{\circ} 11^{\prime} 32^{\prime \prime}$ de latitude Nord. Celle de Tanda est située entre $3^{\circ} 17^{\prime} 10,77^{\prime \prime}$ et $3^{\circ} 16^{\prime} 52,19^{\prime \prime}$ de longitude Est et $12^{\circ} 6^{\prime} 15,35^{\prime \prime}$ et $12^{\circ} 6^{\prime} 42,10^{\prime \prime}$ de latitude Nord. Le climat de la zone est du type soudanien avec deux saisons : une saison sèche (6 à 7 mois) et une saison de pluies (6 à 5 mois). La saison pluvieuse s'étend du mois de mai au mois d'octobre, avec un cumul pluviométrique de plus de $750 \mathrm{~mm}$ (Saadou et al., 2005). La végétation est 
constituée de formations variées allant des forêts (Claire, sèche et galerie) à la savane (Baina, 2000).

La commune rurale de Tamou est localisée dans le département de Say (Région de Tillabery). Elle est située entre $11^{\circ} 54^{\prime}$ et $12^{\circ} 35^{\prime}$ de latitude Nord et $02^{\circ} 4^{\prime}$ et $02^{\circ} 50$ 'de longitude Est (Djibo, 2004). La moyenne pluviométrique annuelle calculée sur 28 ans d'observation (1981-2008) est de $632 \pm 25 \mathrm{~mm}$. Le climat est du type sahélo-soudanien avec deux saisons : une saison sèche de plus longue durée ( 7 à 8 mois) et une saison de pluies de courte durée (4 à 5 mois). La pluviométrie varie de 600 à $800 \mathrm{~mm}$ de pluie par an. En général la végétation de la zone se caractérise par des savanes arborées et arbustives, avec des galeries forestières le long des cours d'eau (Barmo, 2008).

\section{Collecte des données}

Une enquête basée sur les interrogations directes portant sur les usages des plantes médicinales utilisées en pharmacopée traditionnelle et l'impact des techniques de prélèvement sur ces plantes est menée. Il s'agit des informations précises d'une part sur l'informateur, l'identité de la plante, la partie utilisée, le mode de préparation et les usages médicinales et d'autre part sur l'impact des méthodes de prélèvements sur ces ressources. Les interrogations sont directes et basées sur le dialogue en langue locale, accompagnée parfois de l'achat des plantes médicinales vendues ou de la présentation de l'espèce si elle est disponible. Il faut aussi noter qu'un entretien avec des personnes ressources surtout en matière de gestion a été conduit.

\section{Traitement et analyse des données}

Les espèces inventoriées sont identifiées sur le terrain et/ou au laboratoire à l'aide des Flores du Sénégal (Berhaut, 1967 ; 1971 ; 1974 ; 1975 ; 1976 ; 1979), et d'autres ouvrages (Arbres, arbustes et lianes de la zone soudano-sahélienne (Arbonnier 2000), Arbres et arbustes du Sahel. Leurs caractéristiques et leurs utilisations (Maydell, 1983)), mais aussi avec l'aides des enseignants chercheurs de la Faculté des Sciences et Techniques de l'Université Abdou Moumouni de Niamey.

Les maladies recensées ont été regroupées en 14 catégories: Maladies affectant l'appareil circulatoire (CIR), maladies affectant l'appareil respiratoire (RES), maladies affectant l'appareil digestifs (DIG), maladies cutanés et sous-cutanés (CUT), maladies gynéco- obstétriques (GOB), maladies urinaires et génitales (UGE), maladies sensoriels de l'œil (SEN), maladies affectant la gorge (GOR), maladies cardiovasculaires (CAV), maladies osseuses (OS), maladies affectant l'auditifs (AUT), maladies affectant le système nerveux (NER), maladies affectant le système 
endocrinien (END) et les poisons (POI). Ainsi, pour chaque catégorie, le degré de consensus d'utilisation de la plante ou Informant Consensus Factor (ICF) a été calculé suivant la formule de Heinrich et al., (1998) utilisées par plusieurs auteurs (Zerbo et al., (2011); Teklehaymanot et al.,(2009); Tiétiambou et al., (2016); Okullo et al., (2014); Maryo et al., (2015); Shalukoma et al., (2015); Mosaddegh et al., (2016)) et qui s'écrit comme suit:

$$
I C F=\frac{\text { nur }-\mathrm{nt}}{\text { nur }-1}
$$

Avec nur le nombre des mentions des maladies de la catégorie et nt le nombre total de plantes utilisées.

$\mathrm{Au}$ cours de leur investigation sur la perception des espèces en voie de disparition en milieu Gourmantche à l'Est du Burkina Faso, Hahn-Hadjali et Thiombiano (2000) ont révélés que 39 espèces ont été cités comme ayant totalement disparu et 52 espèces comme celles qui sont en voie de disparition et cela dans une période de 20 à 30 ans. Sur la base de cette période (20 à 30 ans), les réponses des personnes âgées de quarante-cinq ans à plus sont les seules prises en compte pour recenser les plantes disparues ou en voie de disparition au cours de cette étude.

Les données recueillies à la suite des enquêtes ont été traitées en utilisant le logiciel tableur Excel 2013 qui, a permis d'établir les histogrammes, mais aussi le calcul des ICF.

\section{Résultats et discussion Analyse floristique}

L'étude a permis de recensr 110 espèces réparties dans 88 genres et 45 familles. Les Fabaceae constituent la famille la plus importante avec 21 espèces, suivie par les Combretaceae avec 5 espèces. Vingt-quatre (24) familles sont représentées chacune par une seule espèce (Tableaux 1 et 2). Les espèces recensées peuvent être classées en herbacées et ligneux. Les herbacées (43 espèces) sont réparties dans 22 familles et 38 genres (Tableau 1) ; et les ligneux (67 espèces) sont repartis dans 25 familles et 51 genres (Tableau 2).

Il ressort du tableau 1 que les familles les mieux représentées en nombre d'espèces sont respectivement celles des Fabaceae (12 espèces), Euphorbiaceae ( 5 espèces), Cucurbitaceae ( 4 espèces) et Chlospermaceae ( 3 espèces). L'analyse du tableau 2, montre que les familles les mieux représentées en nombre d'espèces sont respectivement celles des Fabaceae (15 espèces), Combretaceae (6 espèces), Anarcadiaceae (5 espèces), Rubiaceae (4 espèces), Asclepiadaceae ( 3 espèces), Capparaceae (3 espèces) et Meliaceae (3 espèces). 
Ikhiri et al., (1984) et Saadou (1993) ont recensés respectivement 186 espèces dans les marchés de Niamey et 245 espèces médicinales à l'échelle du territoire Nigérien. Cette différence entre les résultats de l'étude et ceux de Ikhri et al., (1984) s'explique par la différence des lieux d'étude. Pour la différence avec les résultats de Saadou (1993), ceux-ci peut s'expliqué par l'étendue couverte par son étude. Pour avoir le savoir individuel, l'étude a concerné presque toute les couches de la population tandis que ces auteurs se sont adressés à des interlocuteurs bien définis (Tradipraticiens et vendeurs de plantes médicinales).

Tableau 1: Plantes herbacées, organes, usages et mode de préparation

\begin{tabular}{|c|c|c|c|c|}
\hline Familles & Nom scientifique & $\begin{array}{l}\text { Organes } \\
\text { prélevés }\end{array}$ & $\begin{array}{c}\text { Usages médico- } \\
\text { traditionnels }\end{array}$ & $\begin{array}{c}\text { Mode de } \\
\text { préparations }\end{array}$ \\
\hline \multirow{5}{*}{ Acanthaceae } & \multirow{2}{*}{ Blepharis linariifolia } & Feuilles & CIR, DIG & Décoction \\
\hline & & Plante entière & CIR & Décoction \\
\hline & \multirow{3}{*}{$\begin{array}{l}\text { Hygrophila } \\
\text { senegalensis }\end{array}$} & Feuilles & $\begin{array}{c}\text { SEN, AUT, CIR, } \\
\text { END }\end{array}$ & $\begin{array}{l}\text { Macération, } \\
\text { décoction }\end{array}$ \\
\hline & & Fruits & SEN, AUT, CIR & $\begin{array}{l}\text { Macération, } \\
\text { décoction }\end{array}$ \\
\hline & & Tige feuillée & DIG & Décoction \\
\hline Aizoaceae & $\begin{array}{c}\text { Limeum } \\
\text { pterocarpum }\end{array}$ & Plante entière & CIR & Décoction \\
\hline Amaranthaceae & Pupalia lappacea & Plante entière & CIR & Décoction \\
\hline Amaryllidaceae & $\begin{array}{c}\text { Pancratium } \\
\text { trianthum }\end{array}$ & Fruits & OS & Broyage \\
\hline Asteraceae & $\begin{array}{l}\text { Centaurea } \\
\text { perrottetii }\end{array}$ & Plante entière & CUT & Poudre, macération \\
\hline Asparagaceae & Asparagus africanus & Racines & $\mathrm{OS}$ & Décoction \\
\hline \multirow[b]{2}{*}{ Convolvulaceae } & Merremia tridentata & Plante entière & UGE & Poudre, Décoction \\
\hline & Ipomoea asarifolia & Plante entière & $\mathrm{OS}$ & Décoction \\
\hline \multirow{3}{*}{ Chlospermaceae } & \multirow{2}{*}{$\begin{array}{l}\text { Cochlospermum } \\
\text { planchonii }\end{array}$} & Feuilles & CIR & Décoction, poudre \\
\hline & & Racines & CIR & Décoction, poudre \\
\hline & $\begin{array}{c}\text { Cochlospermum } \\
\text { tinctorium }\end{array}$ & Fruits & CIR, UGE & Décoction \\
\hline Capparaceae & $\begin{array}{l}\text { Gynandropsis } \\
\text { gynandra }\end{array}$ & Plante entière & CIR & Décoction \\
\hline Commelinaceae & $\begin{array}{l}\text { Commelina } \\
\text { forskoalei }\end{array}$ & Feuilles & CIR, NER & Décoction, broyage \\
\hline \multirow{6}{*}{ Cucurbitaceae } & \multirow{2}{*}{ Cucumis melo } & Branche & CIR & Décoction \\
\hline & & Racines & CIR & Décoction \\
\hline & Cucumis metuliferus & Fruits & AUT & Poudre \\
\hline & Cucumis metuliferus & Fruits & GOR & Poudre, broyage \\
\hline & $\begin{array}{c}\text { Momordica } \\
\text { balsamina } \\
\end{array}$ & Plante entière & CIR & Décoction \\
\hline & $\begin{array}{l}\text { Chrozophora } \\
\text { brocchiana }\end{array}$ & Feuilles & CIR, NER, DIG & Décoction \\
\hline
\end{tabular}




\begin{tabular}{|c|c|c|c|}
\hline & Fruits & CIR, NER, DIG & Décoction \\
\hline & Racines & CIR, DIG & Décoction, poudre \\
\hline $\begin{array}{l}\text { Euphorbia } \\
\text { aegyptiaca }\end{array}$ & Feuilles & POI & Macération \\
\hline Phyllanthus amarus & Feuilles & DIG & Infusion \\
\hline $\begin{array}{c}\text { Phyllanthus } \\
\text { maderaspatensis }\end{array}$ & $\begin{array}{l}\text { Plante } \\
\text { entière }\end{array}$ & CUT, DIG & Poudre, infusion \\
\hline Flueggea virosa & Feuilles & CAV & $\begin{array}{l}\text { Décoction, } \\
\text { infusion }\end{array}$ \\
\hline $\begin{array}{c}\text { Alysicarpus } \\
\text { ovalifolius }\end{array}$ & $\begin{array}{l}\text { Plante } \\
\text { entière }\end{array}$ & CIR & Décoction \\
\hline Crotalaria pallida & Feuilles & NER & Décoction, poudre \\
\hline & Racines & DIG & Macération \\
\hline & Tiges & NER & Poudre \\
\hline Indigofera tinctoria & Feuilles & CIR & Décoction \\
\hline $\begin{array}{c}\text { Pancratium } \\
\text { trianthum }\end{array}$ & Tubercules & OS & Macération \\
\hline Stylosanthes erecta & Ecorce & CIR & Décoction \\
\hline & Ecorce & CIR & Décoction \\
\hline $\begin{array}{c}\text { Tephrosia } \\
\text { lupunifolia }\end{array}$ & $\begin{array}{l}\text { Plante } \\
\text { entière }\end{array}$ & CIR & Macération \\
\hline Indigofera pilosa & $\begin{array}{l}\text { Plante } \\
\text { entière }\end{array}$ & CIR & Décoction \\
\hline $\begin{array}{c}\text { Tephrosia } \\
\text { pedicellata } \\
\end{array}$ & Ecorce & NER & Décoction, poudre \\
\hline Tephrosia purpurea & Feuilles & $\mathrm{CAV}, \mathrm{POI}$ & Infusion \\
\hline & Racines & $\mathrm{CAV}, \mathrm{POI}$ & Infusion \\
\hline Cassia occidentalis & $\begin{array}{l}\text { Plante } \\
\text { entière }\end{array}$ & CIR & Décoction \\
\hline Cassia italica & Feuilles & CIR & $\begin{array}{l}\text { Décoction, } \\
\text { infusion }\end{array}$ \\
\hline Cassia mimosoides & Racines & DIG & Décoction \\
\hline & $\begin{array}{l}\text { Plante } \\
\text { entière }\end{array}$ & DIG, OS & $\begin{array}{l}\text { Macération, } \\
\text { décoction }\end{array}$ \\
\hline Hyptis suaveolens & Feuilles & CUT & $\begin{array}{c}\text { Feuille fraiche } \\
\text { déposée }\end{array}$ \\
\hline $\begin{array}{c}\text { Tapinanthus } \\
\text { globiferus }\end{array}$ & Feuilles & NER & Poudre \\
\hline Abuliton pannosum & Feuilles & CIR & $\begin{array}{l}\text { Macération, } \\
\text { décoction }\end{array}$ \\
\hline Sesamum alatum & $\begin{array}{l}\text { Plante } \\
\text { entière }\end{array}$ & CIR & $\begin{array}{c}\text { Macération, } \\
\text { décoction }\end{array}$ \\
\hline $\begin{array}{l}\text { Anthephora } \\
\text { nigritana }\end{array}$ & Feuilles & CIR & $\begin{array}{l}\text { Macération, } \\
\text { décoction }\end{array}$ \\
\hline & Racines & DIG & $\begin{array}{l}\text { Macération, } \\
\text { décoction }\end{array}$ \\
\hline
\end{tabular}




\begin{tabular}{cccc} 
Eragrostis tenella & Feuilles & CUT & Poudre, décoction \\
\cline { 1 - 3 } Sterculia setigera & Ecorce & CIR, POI & Décoction, poudre \\
\hline Waltheria indica & Fruits & CUT & Broyer, poudre \\
\hline Striga hermonthica & Feuilles & CIR, CUT, END & Décoction, poudre \\
\hline Racines & CIR, CUT, END & Décoction, poudre \\
\hline Scoparia dulcis & Feuilles & DIG & Décoction, poudre \\
\hline $\begin{array}{c}\text { Schwenkia } \\
\text { americana }\end{array}$ & $\begin{array}{l}\text { Plante } \\
\text { entière }\end{array}$ & CIR & Décoction \\
\hline Typha domingensis & Feuille & CIR & Décoction
\end{tabular}

Tableau 2: Plantes ligneuses, organes, usages et mode de préparation

\begin{tabular}{|c|c|c|c|c|}
\hline Familles & Nom scientifique & $\begin{array}{l}\text { Organes } \\
\text { prélevés }\end{array}$ & $\begin{array}{c}\text { Usages médico- } \\
\text { traditionnels }\end{array}$ & $\begin{array}{c}\text { Mode de } \\
\text { préparations }\end{array}$ \\
\hline \multirow{8}{*}{ Anarcadiaceae } & \multirow{2}{*}{ Heeria insignis } & Branche & CIR & Décoction \\
\hline & & Feuilles & CIR & Décoction \\
\hline & Lannea acida & Ecorce & GOB & Décoction \\
\hline & \multirow{2}{*}{ Lannea microcarpa } & Ecorce & CIR, DIG & Décoction \\
\hline & & Feuilles & DIG & Décoction, macération \\
\hline & Mangifera indica & Feuilles & CIR & Décoction \\
\hline & \multirow{2}{*}{ Sclerocarya birrea } & Ecorce & CIR, DIG & Décoction \\
\hline & & Feuilles & DIG & Décoction \\
\hline \multirow{3}{*}{ Annonaceae } & \multirow{3}{*}{ Annona senegalensis } & Ecorce & CIR, CUT & Décoction, poudre \\
\hline & & Feuilles & CIR & Macération, décoction \\
\hline & & Racines & CIR, POI & $\begin{array}{c}\text { Macération, décoction, } \\
\text { poudre }\end{array}$ \\
\hline \multirow{2}{*}{ Arecaceae } & \multirow{2}{*}{ Hyphaene thebaica } & Feuilles & GOR & Décoction, poudre \\
\hline & & Fruits & NER & Poudre \\
\hline \multirow{8}{*}{ Asclepiadaceae } & \multirow{2}{*}{ Calotropis procera } & Feuilles & GOB & Décoction \\
\hline & & Racines & NER & Poudre, décoction \\
\hline & \multirow{4}{*}{ Leptadenia hastata } & Feuilles & CUT & Poudre, macération \\
\hline & & Plante entière & CUT & $\begin{array}{l}\text { Poudre, macération, } \\
\text { décoction }\end{array}$ \\
\hline & & Fruits & CUT & Poudre, broyage \\
\hline & & Rameau feuillé & DIG & Décoction \\
\hline & \multirow{2}{*}{ Leptadenia pyrotechnica } & Racines & GOB & Décoction \\
\hline & & Tige & GOB & Décoction \\
\hline \multirow{2}{*}{ Balanitaceae } & \multirow{2}{*}{ Balanites aegyptiaca } & Ecorce & CIR, CUT, NER & Décoction \\
\hline & & Racine & CIR, POI & Décoction, poudre \\
\hline \multirow{4}{*}{ Bignoniaceae } & \multirow{3}{*}{ Kigelia africana } & Ecorce & CIR, DIG & Décoction, poudre \\
\hline & & Feuilles & CIR, UGE & Décoction \\
\hline & & Racines & CIR & Décoction, poudre \\
\hline & $\begin{array}{c}\text { Stereospermum } \\
\text { kunthianum }\end{array}$ & Ecorce & CIR & Décoction, poudre \\
\hline \multirow{2}{*}{ Bombacaceae } & \multirow{2}{*}{ Adansonia digitata } & Ecorce & NER, DIG & Macération, poudre \\
\hline & & Feuilles & NER, DIG & Macération, poudre \\
\hline
\end{tabular}




\begin{tabular}{|c|c|c|c|c|}
\hline & \multirow{3}{*}{ Bombax costatum } & Ecorce & CIR & Décoction \\
\hline & & Feuilles & CIR & Décoction \\
\hline & & Racines & $\mathrm{OS}$ & Décoction \\
\hline Burseraceae & Commiphora africana & Ecorce & CIR, NER & Décoction, poudre \\
\hline \multirow{5}{*}{ Capparaceae } & Boscia angustifolia & Ecorce & NER, DIG & Poudre, décoction \\
\hline & \multirow{2}{*}{ Boscia senegalensis } & Fruits & CIR & Décoction \\
\hline & & Racines & GOB & Décoction, poudre \\
\hline & \multirow{2}{*}{ Maerua crassifolia } & Ecorce & $\mathrm{OS}$ & Décoction \\
\hline & & Feuilles & RES & Poudre \\
\hline Caricaceae & Carica papaya & Feuilles & CIR & Décoction \\
\hline \multirow[t]{12}{*}{ Combretaceae } & Anogeissus leiocarpus & Ecorce & $\begin{array}{l}\text { CIR, DIG, SEN, } \\
\text { RES }\end{array}$ & Poudre, décoction \\
\hline & & Feuilles & CIR & Décoction \\
\hline & & Racines & CIR & Décoction \\
\hline & Combretum glutinosum & Ecorce & CIR, DIG, POI & Décoction \\
\hline & & Feuilles & CUT & Poudre, broyé \\
\hline & & Racines & NER & Poudre \\
\hline & Combretum micranthum & Feuilles & CIR, GOB & Décoction, macération \\
\hline & & Racines & CIR, POI & Décoction, poudre \\
\hline & Combretum nigricans & Feuilles & CIR, DIG & Décoction, macération \\
\hline & & Racines & CIR, DIG & Décoction \\
\hline & Guiera senegalensis & Feuilles & $\begin{array}{c}\text { CIR, CUT, DIG, } \\
\text { NER }\end{array}$ & $\begin{array}{c}\text { Macération, décoction } \\
\text { poudre }\end{array}$ \\
\hline & Terminalia avicennioides & Ecorce & DIG, RES, SEN & Décoction \\
\hline \multirow[t]{3}{*}{ Ebenaceae } & Diospyros mespiliformis & Ecorce & CIR, CUT & Décoction, poudre \\
\hline & & Feuilles & CIR & Décoction \\
\hline & & Racines & CIR & Décoction \\
\hline \multirow[t]{18}{*}{ Fabaceae } & Bauhinia rufescens & Ecorce & CIR, DIG & Décoction \\
\hline & & Feuilles & DIG & Macération, décoction \\
\hline & Cassia siamea & Feuilles & CIR, GOB & Décoction \\
\hline & Cassia sieberiana & Ecorce & CIR, DIG & Décoction \\
\hline & & Feuilles & DIG & Décoction \\
\hline & & Racines & CIR, DIG, UGE & Décoction \\
\hline & Detarium microcarpum & Racines & CIR & Décoction \\
\hline & Piliostigma reticulatum & Ecorce & $\begin{array}{l}\text { CIR, DIG, CUT, } \\
\text { RES, OS, GOB }\end{array}$ & Poudre, décoction \\
\hline & & Feuilles & CIR, RES, DIG & Poudre, décoction \\
\hline & Tamarindus indica & Feuilles & CIR & Décoction \\
\hline & Pterocarpus erinaceus & Racines & CIR, DIG, CUT & Décoction, poudre \\
\hline & & Ecorce & CIR, DIG, CUT & Décoction, poudre \\
\hline & Acacia nilotica & Ecorce & RES, DIG & Décoction \\
\hline & & Fruits & CIR, DIG, CUT & $\begin{array}{c}\text { Macération, décoction, } \\
\text { poudre }\end{array}$ \\
\hline & Acacia senegal & Fruits & CIR, CUT & $\begin{array}{c}\text { Macération, décoction, } \\
\text { poudre }\end{array}$ \\
\hline & Acacia sieberiana & Ecorce & DIG & Décoction \\
\hline & & Feuilles & DIG & Macération, décoction \\
\hline & Dichrostachys cinerea & Ecorce & GOB & Décoction \\
\hline
\end{tabular}




\begin{tabular}{|c|c|c|c|c|}
\hline & & Racines & GOB & Décoction \\
\hline & \multirow[t]{2}{*}{ Faidherbia albida } & Ecorce & CIR & Décoction \\
\hline & & Feuilles & CIR & Décoction, macération \\
\hline & Mimosa pigra & Feuilles & DIG & $\begin{array}{l}\text { Décoction, poudre, } \\
\text { macération }\end{array}$ \\
\hline & \multirow[t]{2}{*}{ Parkia biglobosa } & Ecorce & CIR, CUT ORL & Décoction, poudre \\
\hline & & Feuilles & CIR & Décoction \\
\hline & \multirow[t]{2}{*}{ Prosopis africana } & Ecorce & CIR, DIG & Décoction, poudre \\
\hline & & Feuilles & CIR, DIG & Décoction, poudre \\
\hline \multirow[t]{4}{*}{ Meliaceae } & Azadirachta indica & Feuilles & CIR & Macération, décoction \\
\hline & Khaya senegalensis & Ecorce & CIR, DIG, NER & Décoction \\
\hline & Pseudocedrela Kotschyi & Ecorce & CIR & Décoction \\
\hline & & Racines & CIR, DIG & Décoction \\
\hline \multirow[t]{5}{*}{ Moraceae } & Ficus platyphylla & Ecorce & CIR, DIG & Décoction \\
\hline & & Feuilles & UGE & Décoction, poudre \\
\hline & & Fruits & UGE & Décoction, poudre \\
\hline & Ficus sycomorus & Feuilles & CIR, DIG & Décoction \\
\hline & & Ecorce & CIR, DIG & Décoction \\
\hline \multirow[t]{2}{*}{ Moringaceae } & Moringa oleifera & Feuilles & CIR & Décoction \\
\hline & & Graines & END & Poudre, mâché \\
\hline \multirow[t]{2}{*}{ Myrtaceae } & $\begin{array}{c}\text { Eucalyptus } \\
\text { camaldulensis }\end{array}$ & Feuilles & CIR, GOB & Décoction \\
\hline & Psidium guajava & Feuilles & $\mathrm{CIR}$ & Décoction \\
\hline \multirow[t]{2}{*}{ Olacaceae } & Ximenia americana & Feuilles & CIR, GOR, GEN & $\begin{array}{l}\text { Macération, décoction, } \\
\text { poudre }\end{array}$ \\
\hline & & Racines & CIR, GOR, UGE & Décoction, poudre \\
\hline \multirow[t]{2}{*}{ Polygalaceae } & $\begin{array}{c}\text { Securidaca } \\
\text { longipedunculata }\end{array}$ & Ecorce & CIR, OS & Décoction \\
\hline & & Racines & $\begin{array}{c}\text { CAV, CIR, NER, } \\
\text { RES }\end{array}$ & Décoction \\
\hline \multirow[t]{3}{*}{ Rhamnaceae } & Ziziphus mauritiana & Ecorce & CUT & Poudre \\
\hline & & Racines & CIR, DIG, UGE & Décoction, poudre \\
\hline & Ziziphus mucronata & Racines & CIR & Décoction \\
\hline \multirow[t]{5}{*}{ Rubiaceae } & Crossopteryx febrifuga & Feuilles & CIR & Décoction, macération \\
\hline & & Racines & CIR & Décoction \\
\hline & Gardenia erubescens & Fruits & CIR & Décoction \\
\hline & Gardenia sokotensis & Feuilles & CIR & Décoction \\
\hline & Gardenia ternifolia & Ecorce & SEN & Macération \\
\hline \multirow[t]{2}{*}{ Rutaceae } & Citrus aurantifolia & Feuille & CIR, GOB & Décoction \\
\hline & Citrus limon & Feuille & CIR, GOB & Décoction \\
\hline \multirow[t]{3}{*}{ Sapotaceae } & Vitellaria paradoxa & Ecorce & CIR & Décoction \\
\hline & & Racines & GOB & Décoction \\
\hline & & Feuilles & GOB & Décoction \\
\hline \multirow[t]{2}{*}{ Verbenaceae } & Vitex doniana & Ecorce & CIR; CUT & Décoction, macération \\
\hline & & Feuilles & CIR & Décoction \\
\hline \multirow[t]{3}{*}{ Vitaceae } & Ampelocissus africana & Plante entière & OS & Poudre, décoction \\
\hline & & Racines & OS & Poudre, décoction \\
\hline & Cissus populnea & Racines & OS & Décoction, poudre \\
\hline
\end{tabular}




\section{Parties et organes utilisées}

Les organes des plantes utilisées en pharmacopée sont principalement les feuilles avec 35\%, puis viennent les racines $(21,42 \%)$ et les écorces $(21,42 \%)$ (Figure 2). Cette utilisation plus importante des feuilles des plantes médicinales par rapport aux autres organes, confirment les résultats de Salhi et al., (2010), Tahri et al., 2012, Diatta et al., (2013) et de Mosaddegh et al., (2016).

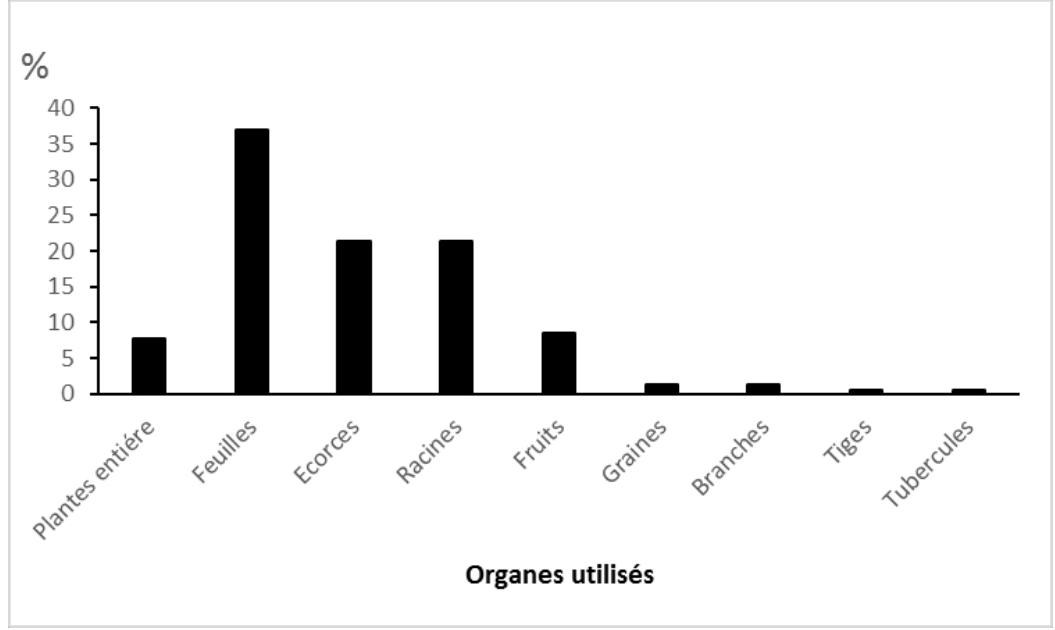

Figure 2 : Proportion des différents organes des plantes utilisées

\section{Techniques de récolte des organes et leur impacts}

Il n'existe pas de réglementation de la cueillette des organes des plantes. Pour les techniques de prélèvement des différentes parties des plantes, plusieurs techniques de récolte sont utilisées par les populations locales en fonction de la partie qu'elles soient bonnes ou mauvaises pour la gestion des plantes.

\section{- Les fruits}

Les fruits qui sont utilisés en pharmacopée sont ramassés une fois tombé. Certaines personnes enquêtées grimpent sur les arbres pour cueillir et/ou couper la branche entière quand les fruits ne sont pas accessibles. D'autres, utilisent une longue tige pour faire tomber les fruits.

\section{- Les écorces}

Pour prélever l'écorce, il faut se munir d'un coupe-coupe pour couper la quantité que l'on veut, sans se soucier de la plante. Ce type de prélèvements est effectué plusieurs fois sur la plante laissant cette dernière avec des séquelles (Apparition de creux, frottures, gouttières, bourrelets) qui ne sont 
pas sans conséquence sur la plante. Selon Traoré et al., (2011) l'écorçage permanent est souvent responsable des troubles physiologiques. Ces derniers ont des impacts sur la production fruitière des espèces causant le problème de disponibilité des semences qui assurent le renouvellement.

\section{- Les racines}

Le prélèvement des racines nécessite la réalisation des trous pour les atteindre, et couper la quantité voulue. Pour les herbacées, certains arbustes et les arbres juvéniles c'est la plante entière qui est prélevée, la racine est coupée et les autres organes abandonnée dans la nature. Le prélèvement des racines porte préjudice à la flore. Il les expose aux aléas climatiques, au dépérissement voire même à la mort, contribuant ainsi à une perte des espèces ou à la disparition de celles en voie de disparition.

Toutes les personnes enquêtées $(100 \%)$ reconnaissent à l'unanimité que cette pratique est la plus destructrice. La meilleure technique pour la récolte des racines consiste à prélever les petites racines et laisser la racine principale ainsi que les racines secondaires.

\section{- Les feuilles}

En ce qui concerne les feuilles, le prélèvement se fait sans technique appropriée. Selon Bitsindou, (1986), la fréquence d'utilisation élevée de feuilles peut être expliquée par l'aisance et la rapidité de la récolte. Pour prélever les feuilles certaines personnes utilisent des outils (machette, couteau etc...) ; d'autres par contre utilisent la main pour casser les branches sans se soucier de la plante. Et Mahamane (1997), d'ajouter si l'ébranchage est intensif, il peut entraîner la baisse de la production fruitière de l'arbre ce qui se répercute sur la disponibilité en semences. Or, si l'espèce est rare ou en danger de disparition, cela conduit inévitablement à sa disparition du fait du manque de semence pour perpétuer l'espèce.

\section{- Rameaux, tiges et branches}

Pour les rameaux et tiges, en général c'est la plante entière qui est prélevée ou les jeunes pousses pour les arbres et arbustes. Après, on prend ce qui est nécessaire et le reste est jeté dans la nature. Pour les branches, c'est avec des outils de coupes ou avec la main que se fait le prélèvement. De fois on peut voir les séquelles (Nœuds ou gouttières) des mauvais prélèvements sur les plantes.

Ces pratiques ne sont pas en adéquation avec la bonne gestion, car pouvant avoir un impact sur l'avenir des espèces. 


\section{- La plante entière}

Plusieurs espèces (Tableau 1) sont concernées par cette technique de prélèvements de la plante entière. Pour certaines espèces telles que les herbacées, c'est la plante entière qui est prélevée pour être utilisée directement où conserver pour une utilisation future.

Quant à l'impact de la cueillette des plantes médicinales sur l'environnement, les racines sont récoltées sans souci d'assurer la survie des plantes ressources. Leur collecte à grande échelle menace les écosystèmes. Le prélèvement d'écorce cause préjudice à certains pieds (Makumbelo et al., 2008). Ces mauvaises pratiques sont inadaptées à la gestion durable des plantes médicinales du fait qu'elles contribuent à leurs destructions. Et selon De Geus (1995), les problèmes pouvant survenir à cause de la cueillette commerciale d'espèces en forêt comprennent: réduction des populations, maintien de la biodiversité, impact sur la productivité et le patrimoine génétique des espèces, impact sur les espèces rares ou vulnérables, dégradation potentielle des écosystèmes forestiers, compaction et dégradation des sols, impacts sur la faune, conflits entre les cueilleurs amateurs et les cueilleurs professionnels, conflits avec la sylviculture et récolte dans des lieux protégés. Ainsi pour les herbacées et les jeunes plants des ligneux, c'est la plante entière qui est arrachée à la main ou à l'aide d'outils (machette, houe, daba etc....).

En général, on note que les populations locales n'ont pas reçu de formation sur les techniques de coupe ou mode de prélèvement des différents organes de plantes, et tous veulent avoir cette formation si nécessaire. Les mauvaises pratiques de prélèvement entraînent l'élimination des individus exploités. À cela, il faut ajouter que l'intensité et la fréquence des prélèvements ne permettent pas un renouvellement de la ressource mais contribue plus à sa destruction. Dans l'optique de conserver et de bien gérer la flore médicinale, il ressort de l'enquête que des mesures doivent être prises parmi lesquelles on note: Limiter les prélèvements, former la population aux différentes techniques de prélèvement des différentes parties des plantes à l'image de ce qui a été fait avec le projet PRSSA (Programme de Renforcement des Structures d'Appui à l'Agriculture) dans le cadre de l'agroforesterie des champs de culture et le projet Keita et enfin créer des pépinières d'espèces médicinales en disparition.

\section{Mode de préparation}

Pour l'administration de la drogue, plusieurs modes de préparation sont employés par les utilisateurs à savoir la décoction, l'infusion, la poudre, la macération et le broyage. Ainsi la décoction est de loin le mode le plus utilisé avec 62,3\% suivi de la poudre et de la macération avec respectivement 16,25 et 15,62 \% (Figure 3). Selon Salhi et al., (2010), la 
décoction permet de recueillir le plus de principes actifs et atténue ou annule l'effet toxique de certaines recettes. Avec la décoction comme premier mode de préparation, nos résultats corroborent ceux de plusieurs auteurs : Zerbo et al., (2010) ; Benkhnigue et al., (2011); Mehdioui et al., (2007).

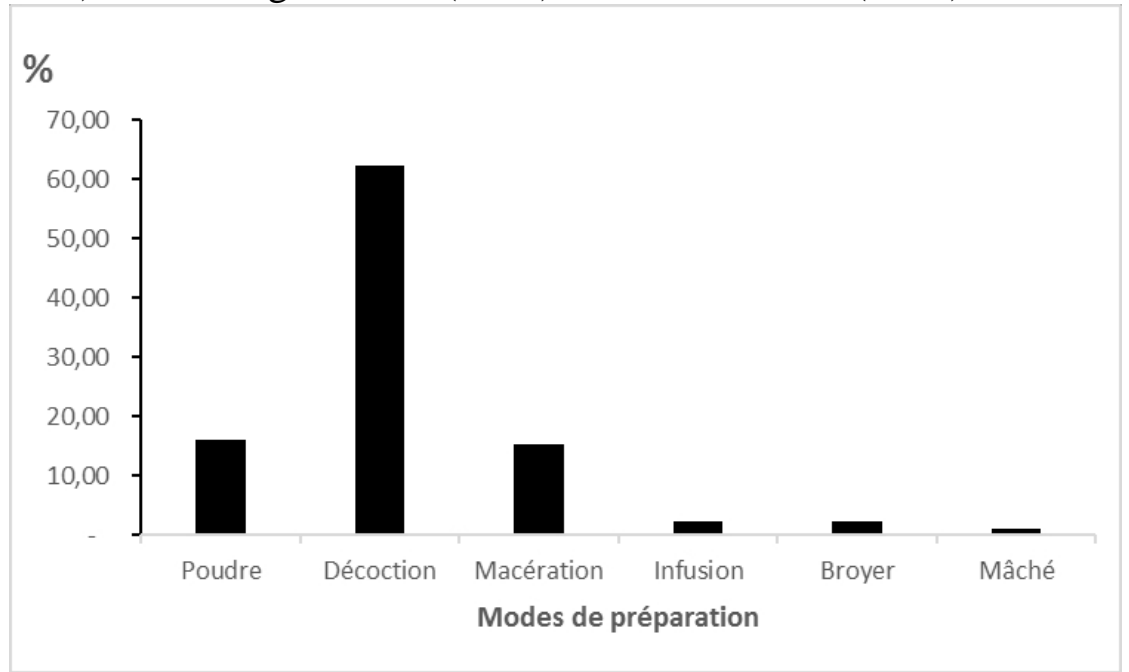

Figure 3 : Proportion des modes de préparation

\section{Phytothérapie et maladies traitées}

Cette étude, a permis de répertorier plusieurs maladies traitées par les plantes médicinales, contribuant ainsi à une meilleure connaissance des soins traditionnels. Ces maladies ont été classées en quatorze catégories (Figure 4). Les résultats obtenus ont montré que la plupart des espèces sont très utilisées dans les soins de l'appareil circulatoire (47,48 \%) (Figure 4). Ces résultats ne sont pas conformes à ceux obtenus par Salhi et al., (2010) et Tahri et al., (2012) qui ont trouvés que les affections digestives arrivent en première position. Ces différences s'expliquent par le fait que les études n'ont pas été conduites dans la même zone, et certaines maladies sévissent en fonction des zones. Selon Pousset (1989), la médecine traditionnelle se justifie en particulier dans le traitement de nombreuses infections que la médecine moderne ne maîtrise pas encore et montre que de nombreuses plantes utilisées telles qu'elles sont aussi efficaces que les médicaments importés par l'Afrique. 


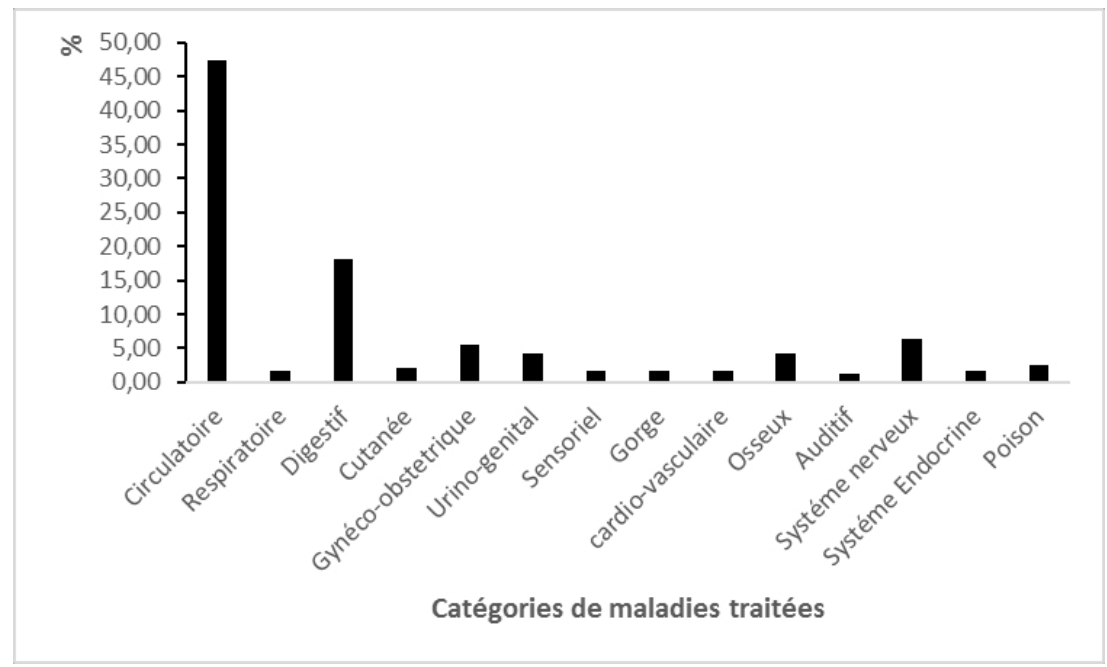

Figure 4 : Proportion des maladies.

Le calcul du degré de consensus d'utilisation (ICF) révèle que toutes les catégories de plantes ont dépassées la moyenne qui est 0,5 (Fig. 5). Les maladies urinaires et génitales ont enregistrées la plus petite valeur $(0,65)$. Maryo et al., (2015) en Ethiopie ont aussi obtenus des indices de degré de consensus élevés. Selon Heinrich et al., (1998), lorsque le facteur de consensus d'informateurs est élevé, il reflète une bonne connaissance des plantes médicinales, un savoir collectif de leurs usages, mais également un échange d'informations entre les tradipraticiens. Pour cette étude l'ICF audessus de la moyenne, implique une bonne connaissance des plantes, mais aussi un échange d'information entre enquêtées, ayant signifiés qu'ils ont appris avec les autres soit à la suite d'une maladie les ayant conduits à des tradipraticiens ou des personnes ayant déjà souffert de la maladie. Très peu ont hérité des connaissances sur les plantes car la majeure partie a obtenu les connaissances d'une manière empirique. Ici ce n'est pas un échange entre tradipraticiens seulement, mais aussi entre personnes de toutes les couches vivant dans les zones rurales. 


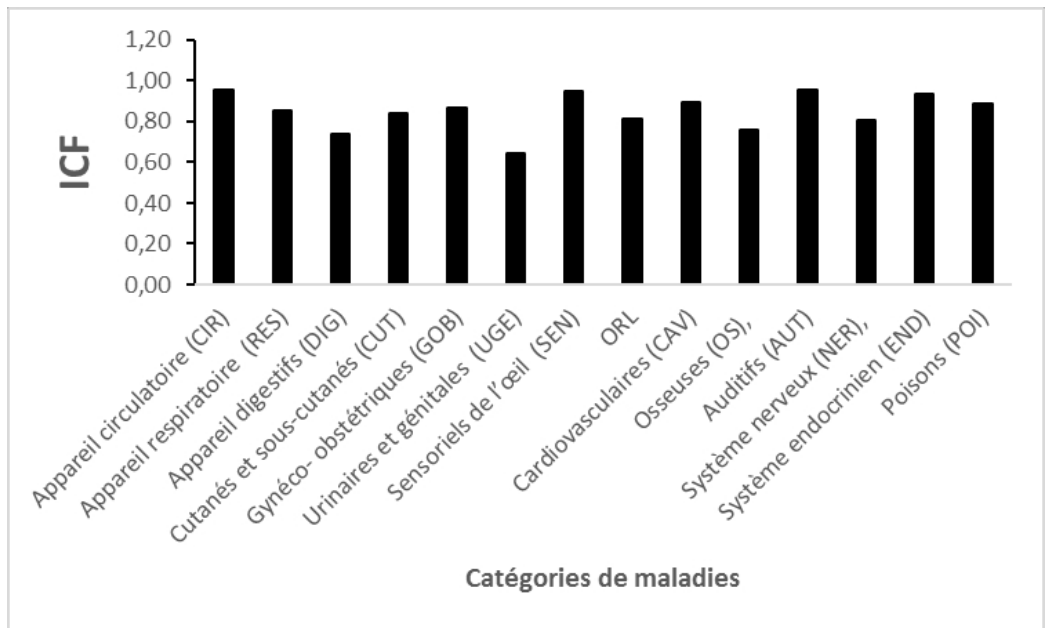

Figure 5 : Degré de consensus d'utilisation (ICF) des plantes.

\section{Accès et lieux d'approvisionnement en plantes médicinales}

À l'unanimité les enquêtés ont répondu que l'accès aux plantes médicinales est libre donc ils n'ont pas besoin d'une quelconque autorisation ni du chef de village et non plus celui des agents forestiers. Pour les lieux d'approvisionnement on note les champs de culture et surtout les forêts de Gorou Bassounga et de Tamou.

Pour Niamey, l'enquête a révélé que les tradipraticiens et vendeurs des plantes médicinales se ravitaillent en grande partie à partir des zones de Gaya et Tamou, qui deviennent ainsi les pourvoyeurs de ces produits pour la communauté urbaine de Niamey. A Gaya et Tanda, $100 \%$ des personnes enquêtés s'approvisionnent en plantes médicinales dans la forêt classée de Gorou Bassounga, pour ceux de Tamou la réserve et le Parc W malgré l'interdiction qui plane sur ces zones.

\section{Commercialisation des espèces médicinales}

$\mathrm{Au}$ village très peu de gens achètent les plantes médicinales. Cependant dans les grandes villes notamment, Niamey et dans les autres villes, la vente des plantes médicinales est effectuée dans les marchés par des personnes des deux sexes où des stands permanents sont installés avec différentes parties (Écorce, feuilles, racines, bourgeons, tige etc....) de plantes. On peut rencontrer aussi des vendeurs et ou vendeuses de plantes médicinales installés dans les quartiers. L'utilisation des plantes médicinales est en vogue, comme le montre la naissance d'une nouvelle forme de commerce de plante médicinale qui se fait actuellement dans la ville de Niamey où on rencontre de plus en plus de marchands ambulants de plantes médicinale. Cette forme de vente se fait à l'aide de charrette (Figure 6) 
rempli de produit de toute sorte (Feuille, écorce, racine, fruit, tige, plante entière etc...).

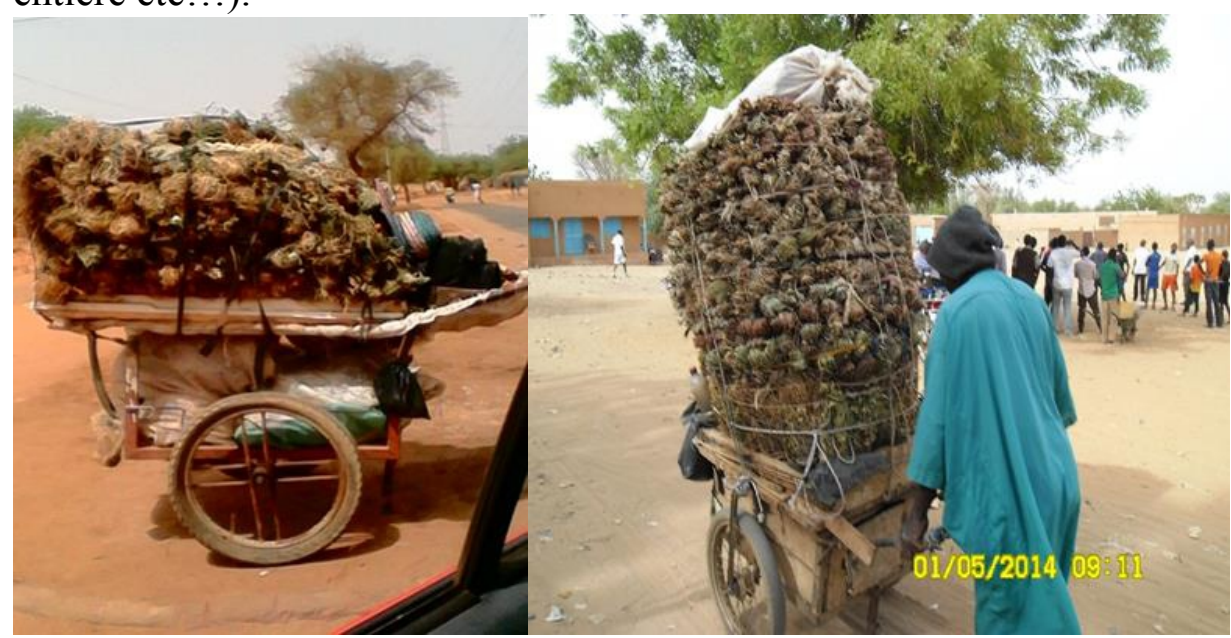

Figure 6 : Vendeurs ambulants de plantes médicinales dans les rues de Niamey

\section{Conservation des produits issus de plantes médicinales}

Les personnes enquêtées ne disposent pas de moyens de conservation des produits finis autre que le séchage. Pour mieux conserver leurs produits certaines personnes rendent les organes en poudre et les conditionnent dans des sachets, des boites ou morceau de tissus. selon Adjanahoun et al., (1988), le seul moyen de conservation est le séchage des organes.

\section{Plantes médicinales disparues ou en voie de disparition}

Vingt-deux espèces végétales réparties dans 11 familles sont déclarées disparues ou en voie de disparition (Tableau 3) selon la population. L'analyse des tableaux 2 et 3 fait ressortir que toutes les espèces disparues ou en voie de disparition ont leurs écorces et ou racines prélevées. Ceci va en adéquation avec les informations des enquêtés qui ont mentionné les prélèvements des parties des plantes comme une des conséquences de la rareté ou disparition des espèces médicinales. Les causes de cette rareté se résument, en plus des prélèvements cités ci haut, aux changements et aléas climatiques, à la coupe de bois (Bois de chauffe, de service et d'œuvre), au surpâturage, aux feux de brousse, à l'augmentation des terres de culture. Toutefois, la population a mentionné que les principales causes de cette rareté ou disparition est la coupe de bois et l'augmentation des terres de culture.

L'analyse du tableau 3 montre que Annona senegalensis $(55,74 \%)$ et Kigelia africana $(63,93 \%)$ sont les seules espèces à avoir une fréquence de citation supérieure à $50 \%$. Ceci est dû certainement par le fait que seules les réponses des personnes ayant un âge supérieur ou égal à 45 ans ont été 
considérées. Parmi les espèces recenses, sept (Adansonia digitata, Detarium microcarpum, Diospyros mespiliformis, Ficus platyphylla, Khaya senegalensis, Parkia biglobosa et Prosopis africana) figurent sur la liste des plantes rares ou disparues de Wezel et Haigis (2000). Six plantes (Boscia senegalensis, Piliostigma reticulatum, Pseusocedrella kotchi, Sclerocarya birrea, Ziziphus mauriatanna et Vitex doniana) de l'étude ne figurent pas sur la liste des espèces végétales rares ou disparues de Hassane (2008).

Les plantes rares ou disparues représentent $24 \%$ du nombre de plantes médicinales recensées dans les sites au cours de cette étude.

Tableau 3 : Plantes médicinales disparues ou en voie de disparition selon la population

\begin{tabular}{ccc} 
Nom scientifique & Familles & Fréquence \% \\
\hline Annona senegalensis & Annonaceae & 55,74 \\
Anogeissus leiocarpus & Combretaceae & 49,18 \\
Bombax constatum & Bombacaceae & 18,03 \\
Boscia senegalensis & Capparaceae & 24,59 \\
Detarium microcarpum & Caesalpiniaceae & 3,28 \\
Diospyros mespiliformis & Ebenaceae & 11,48 \\
Ficus platyphylla & Moraceae & 32,79 \\
Ficus sycomorus. & Moraceae & 40,98 \\
Khaya senegalensis & Meliaceae & 27,87 \\
Kigelia africana & Bignoniaceae & 63,93 \\
Lannea microcarpa & Anacardiaceae & 19,67 \\
Parkia biglobosa & Mimosaceae & 24,59 \\
Piliostigma reticulatum & Caesalpiniaceae & 26,23 \\
Prosopis africana & Mimosaceae & 6,56 \\
Pseusocedrella kotchi & Meliaceae & 11,48 \\
Pterocarpus erinaceus & Fabaceae & 13,11 \\
Sclerocarya birrea & Anacardiaceae & 13,11 \\
Securidaka longepedunculatta & Polygalaceae & 24,59 \\
Tamarindus indica & Caesalpiniaceae & 29,51 \\
Vitellaria paradoxa & Sapotaceae & 9,84 \\
Ziziphus mauritiana & Loganiaceae & 13,11 \\
Vitex doniana & Verbenaceae & 14,75 \\
\hline & &
\end{tabular}

\section{Gestion durable des plantes médicinales}

La gestion des plantes médicinales passe par la conservation et une utilisation rationnelle, et pour cela dans les villages enquêtés, $52 \%$ conservent les espèces dans les champs à cause du rôle médicinal qu'elles jouent. On note cependant que les populations locales ne plantent pas d'arbres, sauf dans les concessions où ils arrivent à planter ces arbres. Dans les champs de culture, ils conservent les jeunes plantes à cause de leur rôle (Alimentaire, médicinal, fourrager etc.). Il faut noter que dans le cadre de la conservation des plantes médicinales, l'association des tradipraticiens du 
Niger (ATPN) s'est octroyée sur fond propre 12 parcelles situées à la périphérie de la communauté urbaine de Niamey. Cet espace servira comme espace vert ou seront planté les espèces médicinales disparues ou en voie de disparition.

Les solutions envisagées pour contribuer à la conservation et à la gestion durable des plantes médicinales citées par la population locale se résument comme suit :

- la sensibilisation des populations en vue de la conservation et de la culture des plantes médicinales;

- la vulgarisation des informations sur les plantes médicinales ;

- la formation sur les techniques appropriées de prélèvement des plantes médicinales ;

- la mise en place de pépinières villageoises de plantes spontanées disparues ou en voies de disparition.

\section{Conclusion}

Les résultats obtenus lors de cette étude, ont permis de rassembler plusieurs informations sur les espèces médicinales utilisées par la population dans divers traitements de maladies. Divers organes (feuilles, fleurs, fruits, racines, tiges) et des plantes entières, provenant de 110 espèces végétales sont utilisés par les populations pour les traitements des maladies. Cinq modes de préparations traditionnelles des médicaments (décoction, infusion, macération, poudre, broyer, mâcher) ont été enregistrés. Le prélèvement des racines, est le mode le plus destructeur des espèces médicinales selon les personnes enquêtées.

Cette étude constitue une contribution au recensement des plantes médicinales utilisées par la population pour des soins, mais a aussi permis d'évaluer l'impact des prélèvements des organes sur la survie des plantes vue par la population elle-même, qui a proposée des solutions. La culture des plantes médicinales, la réglementation de la récolte des plantes et la formation des populations sur les méthodes de prélèvements des différentes parties des plantes pourraient réduire la pression sur les espèces médicinales et assurer leur pérennité pour une gestion durable.

\section{References:}

1. Adjanohoun E., 1988. Contribution aux études ethnobotaniques et floristiques en république populaire du Congo. ACCT. Paris. 605p.

2. Arbonnier, M., 2000. Arbres, arbustes et laines des zones sèches d'Afrique de l'Ouest. CIRAD-

3. MNHN-UICN. $2^{\text {ème }}$ édition. 573 p.

4. Baina, D., 2000. Contribution à l'étude floristique, écologique et phytosociologique de la forete classée de Gorou Bassounga et des 
milieux cultivés adjacents. Thése de Doctorat de 3e cycle de Biologie et Ecologie Végétales. Université Abdou Moumouni de Niamey. $151 \mathrm{p}$.

5. Barmo S., 2008. Analyse socio-économique de l'exploitation des ressources végétales de la Reserve Totale de Faune de Tamou (RTFT)-Niger. Mémoire de DEA. Biologie. Faculté des Sciences et Techniques. Université Abdou Moumouni de Niamey. 67p.

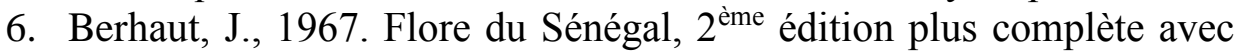
les forêts humides de la Casamance. $485 \mathrm{p}$.

7. Berhaut, J., $1971 ; 1974 ; 1975 ; 1976 ; 1979$. Flore illustrée du Sénégal. Dicotylédones. 6 volumes.

8. Bitsindou, M., 1986. Enquête sur la phytothérapie traditionnelle à Kindamba et Odzala (Congo) et analyse de convergence d'usage des plantes médicinale en Afrique centrale. Mem. Doc (inéd.). Univ. Libre de Bruxelles. 482p.

9. Dasylva, B., 2001. Contribution à l'étude de l'herboristerie traditionnelle sénégalaise : Inventaire des plantes médicinales vendues dans les marchés de Dakar et contrôle de qualité sur 170 échantillons. Thèse de Doctorat. Université Cheik Anta Diop, Dakar, Sénégal.

10. De Geus. P.M., 1995. Botanical Forest Products in British Columbia. An Overview. Integrated Resources Policy Branch. British Columbia Ministry of Forests. 51p.

11. Dibong, S, D., Mpondo Mpondo, E., Ngoye A., Kwin, M, F., 2011. Plantes médicinales utilisées par les populations Bassa de la région de Douala au Cameroun, Int. J. Biol. Chem. Sci. 5(3): 1105-1117.

12. Diatta, C, D., Gueye, M., Akpo, L, E., 2013. Les plantes médicinales utilisées contre les dermatoses dans la pharmacopée Baïnounk de Djibonker, région de Ziguinchor (Sénégal). Journal of Applied Biosciences 70: 5599- 5607.

13. Djibo, M., 2004. Intégration des populations riveraines dans la gestion du Parc National du W du Niger (Niger). Mémoire DESS, Filière: Gestion de la Faune. Université de Liège, Belgique, 66p.

14. Hahn-Hadjali, K., Thiombiano, A., 2000. Perception des espèces en voie de disparition en milieu gourmantche (Est du Burkina Faso). Berichte des Sonderforschungsbereichs 268. Band 14. Frankfurt a.M 285-297.

15. Hassane, H., 2008. Répertoire des espèces végétales les plus couramment utilisées en pharmacopée traditionnelle et impact des techniques de prélèvement sur la diversité biologique dans la réserve de Biosphère du $\mathrm{W}$ du Niger. Mémoire de DEA. Géographie. Faculté 
des lettres et des sciences humaines. Université Abdou Moumouni. $113 \mathrm{p}$.

16. Heinrich, M., Ankli, A., Frei, B., Weimann, C, Sticher, O., 1998. Medicinal Plants in Mexico: Healer's Consensus and Cultural Importance. Social Science and Medecine, 47: 1859-1871.

17. Ikhiri, K., Garba, M., Saadou, M., 1984. Pharmacopée traditionnelle: Recherche sur la pharmacopée au Niger, 5 p.

18. Mahamane, A., 1997. Structure, fonctionnement et dynamique des parcs agroforestiers dans l'Ouest du Niger. Thèse de Doctorat 3ème Cycle. Université de Ouagadoogou. 215 pages.

19. Makumbelo, E., Lukoki, L., Paulus, J, J., Luyindula, N., 2008. Stratégie de valorisation des espèces ressources des produits non ligneux de la savane des environs de Kinshasa: II. Enquête ethnobotanique (aspects médicinaux). Tropicultura, 26, 3, 129-134.

20. Manzo, M., 1996. Etude des jachères dans l'ouest du Niger. Gestion traditionnelle et structurale du peuplement végétal dans le canton de Torodi. Thèse de troisième cycle, Université de Ouagadougou (Burkina Faso), 117 p.

21. Mosaddegh, M., Esmaeili, S., Hassanpour, A, S., Malekmohammadi, M., Naghibi, F., 2016. Ethnobotanical study in the highland of Alvand and Tuyserkan, Iran. Research Journal of Pharmacognosy, 3(1), 7-17

22. Maryo, M., Nemomissa, S., Bekele, T., 2015. An ethnobotanical study of medicinal plants of the Kembatta ethnic group in Ensetbased agricultural landscape of Kembatta Tembaro (KT) Zone, Southern Ethiopia. Asian Journal of Plant Science and Research, 5(7):42-61.

23. Maydell, H, J, V., 1983. Arbres et arbustes du Sahel. Leurs caractéristiques et leurs utilisations. Eschborn (Allemagne) G.T.Z.,Schriftenreihe, 353p.

24. Mehdioui, R., Kahouadji, A., 2007. Etude ethnobotanique auprès de la population riveraine de la forêt d'Amsittène : cas de la Commune d'Imi n'Tlit (Province d'Essaouira). Bulletin de l'Institut Scientifique, Rabat, section Sciences de la Vie, n²9, 11-20.

25. Mounkaila, S., Soukaradji, B., Abdoulaye, A., Mahamane, A., Ikhiri, K., Morou, B., Karim, S., 2015. Essai de germination et de croissance de Artemisia annua L. au Niger. Int. J. Biol. Chem. Sci. 9(1): 108120.

26. OMS (rapport Organisation mondiale de la Santé Genève) No 2 mai 2002. Médecine Traditionnelle : Besoins Croissants et Potentiel.

27. Okullo, J, B, L., Omujal, F., Bigirimana, C., Isubikalu, P., Malinga, M., Bizuru, E., Namutebi, A., Obaa, B, B., Agea, J, G., 2014. Ethno- 
Medicinal Uses of Selected Indigenous Fruit Trees from the Lake Victoria Basin Districts in Uganda. Journal of Medicinal Plants Studies, Vol. 2.

28. Ouôba, P., Lykke, A. M., Boussim J., Guinko, S., 2006. La flore médicinale de la Forêt Classée de Niangoloko (Burkina Faso). Etudes flor. vég. Burkina Faso, 10, 5-16.

29. Pousset, J, L., 1989. Plantes médicinales africaines. Utilisation Pratique. $2^{\text {ième }}$ Tome, ACCT, Paris, $156 \mathrm{p}$.

30. Saadou, M., 1993. Les plantes médicinales du Niger: premier supplément à l'enquête ethnobotanique de 1979. Revue Méd. Pharm. Afr., Vol. 7, No 1 pp 11:24.

31. Saadou, M., 2005. Rapport scientifique de l'observatoire de FalmeyGaya. Niamey. Niger.82p

32. Salhi, S., Fadli, M., Zidane, L., Douira, A., 2010. Études floristique et ethnobotanique des plantes médicinales de la ville de Kénitra (Maroc). Lazaroa 31: 133-146.

33. Shalukoma, C., Bogaert, J., Duez, P., Stévigny, C., Pongombo, C., Visser, M., 2015. Les plantes médicinales de la région montagneuse de Kahuzi-Biega en République Démocratique du Congo : utilisation, accessibilité et consensus des tradipraticiens. Bois et Forêts des Tropiques, $\mathrm{N}^{\circ} 326$ (4). 43-55.

34. Tahri, N., EL Basti, A., Zidane, L., Rochdi, A., Douira, A., 2012. Etude Ethnobotanique des plantes médicinales dans la province De Settat (Maroc). Journal of Forestry Faculty Kastamonu Üni., Orman Fakültesi Dergisi, 12 (2): 192-208.

35. Tabuti, J, R, S., Lye, k.a., Dhillion, S, S., 2003. Traditional herbal drugs of Bulamogi, Uganda: plants, use and administration. J. Ethnopharmacology. 88: 19-44.

36. Teklehaymanot, T., 2009. Ethnobotanical study of knowledge and medicinal plants use by the people in Dek Island in Ethiopia. Journal of Ethnopharmacology 124, 69-78.

37. Traoré, L., Ouédraogo, I., Ouédraogo, A., Thiombiano, A., 2011. Perceptions, usages, et vulnérabilité des ressources végétales ligneuses dans le Sud-Ouest du Burkina-Faso. Int. J. Biol. Chem. Sci., 5(1): 258-278.

38. Tiétiambou, F, R, S., Lykke A, M., Korbéogo, G., Thiombiano, A., Ouédraogo, A., 2016. Perceptions et savoirs locaux sur les espèces oléagineuses locales dans le Kénédougou, Burkina Faso. Bois et forêts des tropiques, $\mathrm{n}^{\circ} 327$.

39. Yamba, B., 1993. Ressources ligneuses et probleme d'amenagement forestier dans la zone agricole du Niger. Thèse de Doctorat, Tome I. Université Michel Montaigne, Bordeaux III, p 29. 
40. Yacouba, H., 1999. Les espèces ligneuses exotiques de la communauté urbaine de Niamey (Niger). Mémoire de DEA, Biologie, Faculté des Sciences et Techniques. Université Abdou Moumouni de Niamey, $83 \mathrm{p}$.

41. Wezel, A., Haigis, J., (2000). Farmer's perception of vegetation changes in semi-arid Niger. Land degradation and development, 11:523-534.

42. Wezel, A., 2002. Plantes médicinales et leur utilisation traditionnelle chez les paysans au Niger. Etudes flor. Vég. Burkina Faso 6, 9-18.

43. Zerbo, P., Millogo-Rasolodimby, J., Nacoulma-Ouedraogo, O. G., Van Damme, P., 2011. Plantes médicinales et pratiques médicales au Burkina Faso : cas des Sanan. Bois et Forêts des Tropiques, 307 (1) : 41-53. 